\title{
Wear of carbide inserts during turning of C45 steel in dry cutting conditions and in presence of emulsion mist
}

\author{
Eugene E. Feldshtein ${ }^{1, *}$, and Szymon Wojciechowski² \\ ${ }^{1}$ University of Zielona Gora, 4 Prof. Z. Szafrana street, 65-516 Zielona Gora, Poland \\ ${ }^{2}$ Poznan University of Technology, Faculty of Mechanical Engineering and Management, Institute of Mechanical Technology, Poznan, \\ Poland
}

\begin{abstract}
This paper describes the wear features of indexable carbide P25 inserts when turning C45 steel with cutting speeds of $250-450 \mathrm{~m} / \mathrm{min}$ in dry cutting conditions and in the presence of an emulsion mist (MQCL method). As the active component of the emulsion mist $5 \%$, the aqueous emulsion of EMULGOL concentrate was used. While analyzing the size, shape and formation dynamics of tool wear, the larger dimensions were found for dry cutting. The statistical analysis of results allowed calculating the dependencies to determine the wear rates. The SEM analysis revealed significant oxygen contents at the contact areas that are due to high temperatures in the cutting zone. When cutting with the emulsion mist the presence of phosphorus and copper entering in EMULGOL concentrate was fixed directly on the wear areas. It suggests the possibility of using compounds included in the emulsion mist to form AW and EP films at the contact areas in the cutting zone.
\end{abstract}

\section{Introduction}

Under present conditions the characteristic factor for the production intensification is the requirement of the productivity growth and reducing the processing cost. This can be achieved, while ensuring efficient operation of the cutting tools and with the use of materials with good machinability. Improved machinability of the material can be achieved in various ways, in particular through effective lubricating and cooling technological mediums and methods for their introduction into the cutting area $[1,2]$.

In latter case, various technical solutions can be used. In particular, the technological fluid can be introduced into the cutting zone with an elevated pressure through the cutting tool or from the outside [2], or the machining is realized in dry or "almost dry" conditions (near dry cutting).

There are two possible ways in this case: the use of oil mist (minimum quantity lubrication, MQL) or emulsion mist (minimum quantity cooling, MQC). Since the particles of oil, soap, various additives are always contained in the emulsion, the method called MQCL (minimum quantity cooling and lubrication) can be discussed.

Cooling methods based on very low amounts of coolants and lubricants, in comparison to dry cutting, enable the reduction of surface roughness [3-5], tool wear [6, 7], cutting force [5, 8], cutting temperature [9] and advantageous chip shape modification, as well as the [10] beneficial stress distribution [11, 12]. Moreover, in order to improve the lubricating conditions of the MQL method, the nanoparticles made of silver, copper and molybdenum disulfide, as well as the carbon nanotubes are being provided $[8,13]$. However, in case of MQCL method, the extreme pressure EP and anti-wear AW additives are being applied $[10,14]$. In these techniques, the oil and emulsion mist formation parameters play an important role. The researches $[15,16]$ revealed that the lower droplets can penetrate the cutting zone in a better way (MQL method), and enable the faster heat dissipation from the cutting zone (MQCL method) [17, $18]$.

During the machining processes, the tool wear is usually manifested by the abrasion wear on the flank face and the crater wear formed onto the rake face $[6$, 19]. Therefore, the prediction of tool wear is necessary to avoid its catastrophic failure, and thus affecting the machining efficiency [20].

Chetan et al. [15] investigated the tool wear of inserts coated with TiN layer during dry and MQCL turning of Nimonic 90 and Ti6Al4V aircraft alloys. The biodegradable emulsion consisting of sunflower oil mixed with water in 10:1 proportions has been used as an active medium. The application of MQCL method caused the lower tool wear in comparison to that obtained during dry turning (especially for Ti6Al4V alloy). This can be attributed to the improved penetration and wettability of machined surface by the active medium's droplets due to increased wetting angle. The wetting angle obtained on the surface of NIMONIC 90 alloy was higher than that obtained on the surface of Ti6A14V alloy, which consequently leads to the

\footnotetext{
* Corresponding author: E.Feldsztein@ibem.uz.zgora.pl
} 
appropriate wetting of surface and formation of lubricating layer.

The aim of this study is to evaluate the effect of the emulsion mist on the character and intensity of the carbide inserts wear when $\mathrm{C} 45$ steel finish turning.

\section{Experimental details}

The C45 EN 10083-2:1991 carbon structural steel was used to evaluate the cutting tool life [21]. Turning was performed using the lathe with CSDBM 2020-M12 toolholder and SNUN120408 insert, made of P25 ISO 513: 2012 cemented carbide.

Turning was performed with a cutting depth of 0.5 $\mathrm{mm}$, feed rate of $0.1 \mathrm{~mm} / \mathrm{rev}$ and cutting speeds in the range of $250-450 \mathrm{~m} / \mathrm{min}$. Insert's wear were analyzed in dry cutting conditions and in the presence of the emulsion mist. As the active component of the emulsion mist, the $5 \%$ aqueous emulsion of EMULGOL concentrate was used.

To determine the chemical composition of the concentrate the MiniPal X-ray spectrophotometer was used which is based on X-ray fluorescence analysis method. The measurement results are shown in Table. 1.

To generate emulsion mist, the Mikronizer $1 \mathrm{LN}$ Micro Unit Wlenox Nozzle device was used [17]. The compressed air pressure was of 70 PSI $(0.48 \mathrm{MPa})$. Emulsion mist was fed to the cutting zone with the three nozzles of $1.2 \mathrm{~mm}$ diameter, wherein the side nozzles were located at an angle of $30^{\circ}$ relative to the central nozzle. The distance from the nozzles plane to the cutting zone was $0.3 \mathrm{~m}$. The compressed air flow was $5.8 \mathrm{l} / \mathrm{min}$ and the emulsion flow was $26.4 \mathrm{~g} / \mathrm{h}$. These conditions are optimal from the point of view of the mist droplet size and conditions of heat removal from the cutting zone [17]. analysis of dynamical properties has been conducted on the three ball end mills with various lengths (see Tab. 1, Fig. 1).

Table 1. The chemical composition of the EMULGOL concentrate.

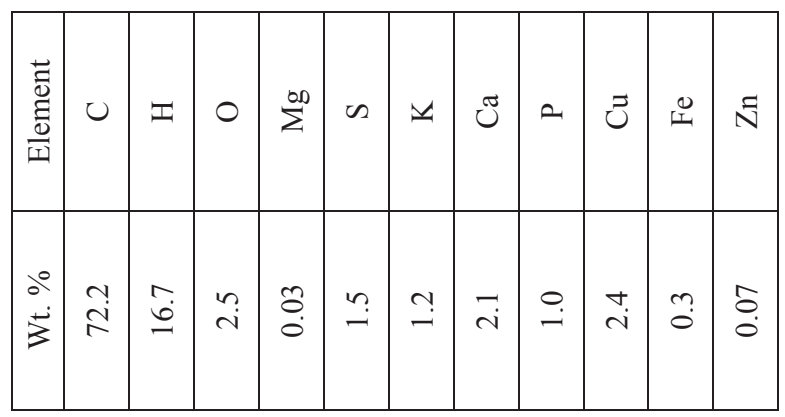

Wear of carbide inserts was estimated with a resolution of $0.01 \mathrm{~mm}$, using an optical microscope with a digital camera after every 4 min, until the critical tool wear has been reached. Limit values of the wear were recorded with the Dino-Lite AM7013MZT universal digital microscope. The 3D images of wear areas were obtained using an IFM 6.1 optical microscope (Infinite Focus Measurement Machine). The SEM studies of worn surfaces were carried out using the JEOL JSM-5600LV microscope with X-ray microanalyzer.

\section{Results and discussion}

The analysis of worn surfaces of carbide inserts on the flank faces, and craters on the rake faces, reveals that their dimensions are greater during dry cutting (Fig. 1). Registered 3D images of worn surfaces confirmed the results of the measurements and revealed a more complex character of inserts wear when dry turning.

The dynamics of carbide inserts wear is illustrated in Fig. 2, which shows the changes in time for the maximum height of wear VBmax of the main flank face. It is easy to notice the advantages for turning in an emulsion mist environment. The Fig. 2 shows that independently on cutting speed value, the tool wear growth is lower during turning in MQCL conditions. In case of turning in the highest investigated cutting speed, the tool wear rate obtained for the MQCL method is 40 $\%$ lower than that reached during the dry turning.

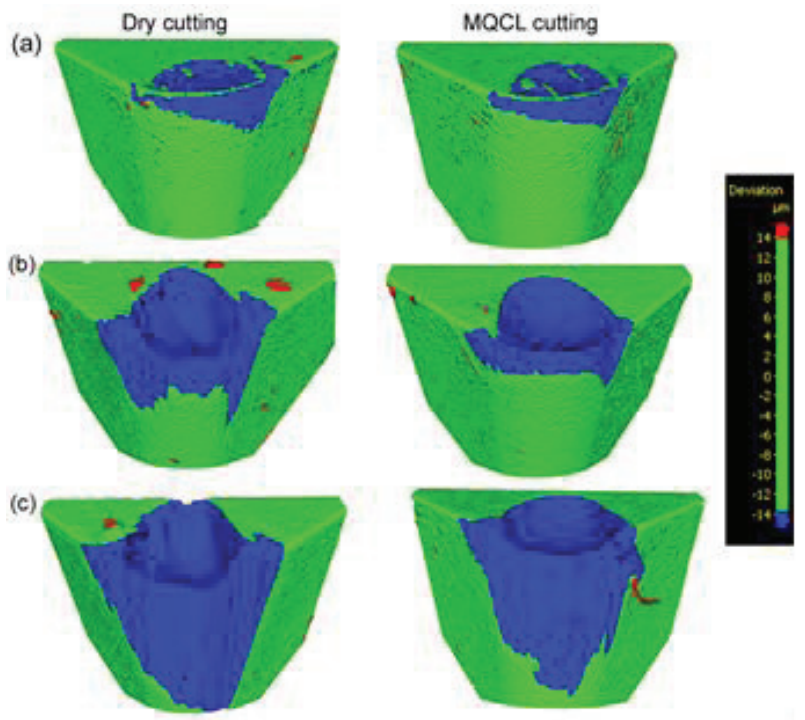

Fig. 1. 3D images of wear areas: a) $v_{c}=250 \mathrm{~m} / \mathrm{min}$; b) $v_{c}=350$ $\mathrm{m} / \mathrm{min}$; c) $v_{c}=450 \mathrm{~m} / \mathrm{min}$.

To determine the causes of the emulsion mist effectiveness, the SEM analysis of the worn surfaces, which include the main flank (Fig. 3) and rake faces (Fig. 4), was carried out. Studies were realized for the cutting speed of $250 \mathrm{~m} / \mathrm{min}$, which is recommended as typical for finish turning of structural steels. The analysis fixed the presence of iron on worn surfaces which indicates the prevalence of the adhesive nature of the wear, as well as high, 16-20\% oxygen content.

It can be assumed that the high temperatures in the cutting zone cause the formation of oxides on contact surfaces. During cutting with the emulsion mist, the presence of phosphorus and copper was recorded directly on the wear areas that are included in the EMULGOL concentrate. These elements are known for their ability to form compounds on the friction surfaces that reduce friction coefficient and wear rate. 

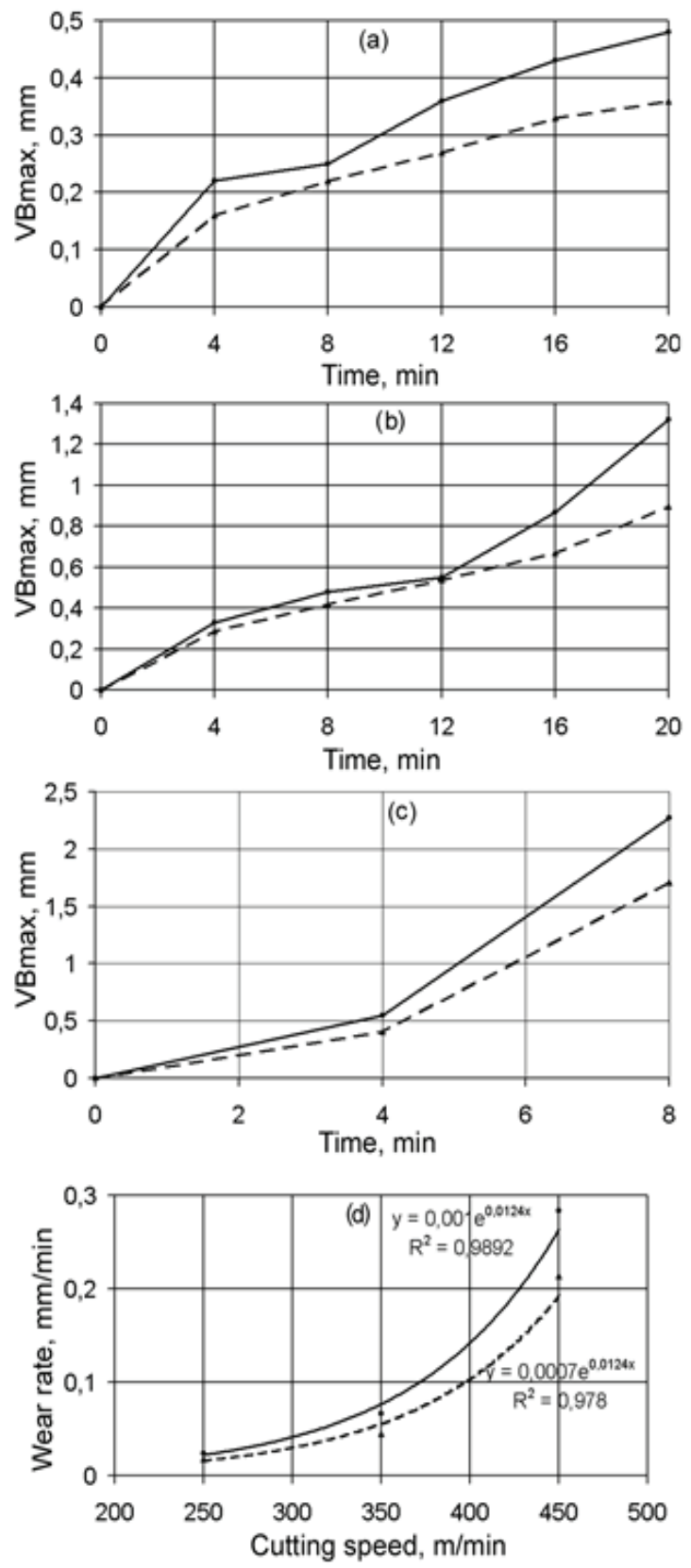

Fig. 2. Wear dynamics $(\mathrm{a}-\mathrm{c})$ and the wear rate $(\mathrm{d})$ for carbide inserts: a) $v_{c}=250 \mathrm{~m} / \mathrm{min}$; b) $v_{c}=350 \mathrm{~m} / \mathrm{min}$; c) $v_{c}=450$ $\mathrm{m} / \mathrm{min}$, continuous line - dry turning, dashed line - MQCL.
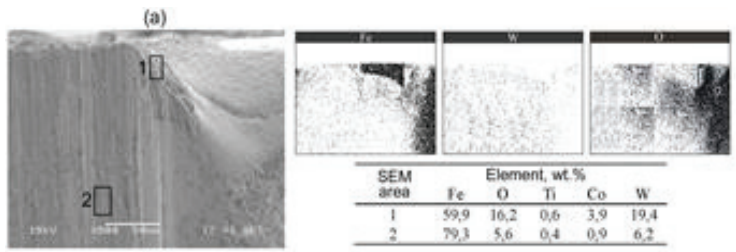

(b)
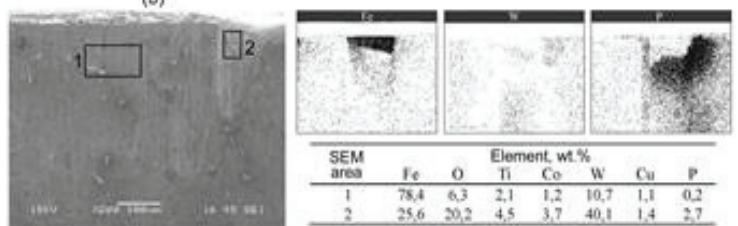

Fig. 3. SEM results for worn areas of the main flank face when cutting speed of $250 \mathrm{~m} / \mathrm{min}$ : a) after dry cutting; b) after MQCL cutting.
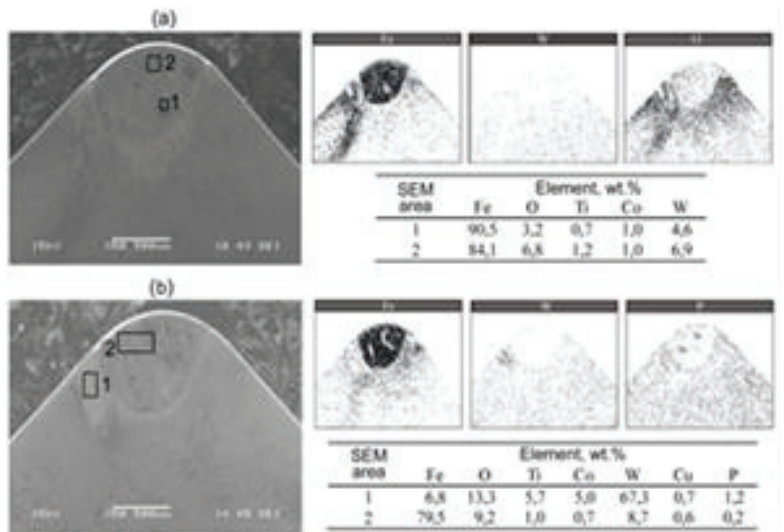

Fig. 4. SEM results for worn areas of the rake face when cutting speed of $250 \mathrm{~m} / \mathrm{min}$ : a) after dry cutting; b) after MQCL cutting

This confirms the possibility of using the compounds included in the emulsion mist to form anti-wear and extreme pressure films on the contact areas in the cutting zone [14].

\section{Conclusions}

Machining of steels in emulsion mist environment has significant advantages over the dry cutting, in particular, in the case of the cutting tools wear rate. In conditions of emulsion mist the dimensions of the wear area are significantly smaller, and this difference is the more, the more there is the cutting speed. Wear rate is decreased by $40 \%$ when cutting with the highest of cutting speeds investigated, while at lower speeds the difference is reduced to $30 \%$. The EDS analysis showed, these positive changes are due to the appearance of active chemical compounds in the cutting area, which are included in the emulsion mist and form anti-wear and extreme pressure films on the contact surfaces. Based on the results obtained, the following general scientific and technological trends can be proposed:

- the maximum efficiency of emulsion mist using is ensured at high cutting speeds,

- the introduction of the active additives in the emulsion mist improves the operating conditions of this method.

\section{References}

1. K. Weinert, I. Inasaki, J.W. Sutherland, T. Wakabayashi, CIRP Ann. - Manuf. Techn. 53, 2 (2004)

2. V.P. Astakhov, Editor. J.P. Davim. Springer-Verlag Limited, London, (2008)

3. R.W. Maruda, S. Legutko, G.M. Krolczyk, C. Lukianowicz, A. Stoic, Teh. Vjes. 22, 5 (2015)

4. G.M. Krolczyk, R.W. Maruda, P. Nieslony, M. Wieczorowski, Measurement, 94, (2016) 
5. V.N. Gaitonde, S.R. Karnik, J.P. Davim, J. Mater. Process. Tech. 204, 1-3 (2008)

6. R.W. Maruda, G.M. Krolczyk, E. Feldshtein, P. Nieslony, B. Tyliszczak, F. Pusavec, Wear 372-373, (2017)

7. N.R. Dhar, M. Kamruzzaman, M. Ahmed, J. Mater. Process. Tech. 172, 2 (2006)

8. Y. Zhang, C. Li, D. Jia, B. Li, Y. Wang, M. Yang, Y. Hou, X. Zhang, J. Mater. Process. Tech. 232, (2016)

9. M. Hadad, B. Sadeghi, Int. J. Mach. Tools Manuf. 63, (2012)

10. R.W. Maruda, G.M. Krolczyk, P. Nieslony, S. Wojciechowski, M. Michalski, S. Legutko, J. Manuf. Processes, 24, (2016)

11. R.W. Maruda, G.M. Krolczyk, M. Michalski, P. Nieslony, S. Wojciechowski J. Mater. Eng. Perform. 26, 1 (2017)

12. H. Hassanpour, M.H. Sadeghi, A. Rasti, S. Shajari, J. Clean. Prod. 120, (2016)

13. P. Krajnik, A. Rashid, F. Pušavec, M. Remškar, A. Yui, N. Nikkam, M.S. Toprak, J. Clean. Prod. 112, (2015)

14. R.W Maruda, S. Legutko, G.M. Krolczyk, S. Hloch, M. Michalski, Int. J. Surf. Sci. Eng. 9, 5 (2015)

15. B. Chetan, C. Behera, S. Ghosh, and P.V. Rao, Ceram. Int. 42, 13 (2016)

16. M.B.G. Jun, S.S. Joshi, R.E. DeVor, S.G. Kapoor, Trans. ASME, J. Manuf. Sci. Eng. 130, 3 (2008)

17. R.W. Maruda, G.M. Krolczyk, E. Feldshtein, F. Pusavec, M. Szydlowski, S. Legutko, A. SobczakKupiec, Int. J. Mach. Tools Manuf. 100, 1 (2016)

18. R.W. Maruda, E. Feldshtein, S. Legutko, G.M. Krolczyk, Teh. Vjes. 22, 5 (2015)

19. G. Poulachon, A. Albert, M. Schluraff, I.S. Jawahir, Int. J. Mach. Tools Manuf. 45, 2 (2005)

20. G.K. Dosbaeva, M.A. El Hakim, M.A. Shalaby, J.E. Krzanowski, S.C. Veldhuis, Int. J. Refract. Met. Hard Mater. 50, (2015)

21. ISO 3685:1993. Tool-life testing with single-point turning tools 